\title{
LA EXPRESIÓN DE LA CAUSA EN HOMERO CON REFERENTES HUMANOS *
}

LUZ CONTI

In the designation of human beings, the Homeric poems show different expressions of Cause, the use of which is determined by their grade of dependence of the verb and by their meaning itself. Their use as the second argument of the verb makes possible the occurrence of the Genitive, the ancient Indoeuropean Ablative, without any preposition; their use as a satellite leads to the occurrence of prepositional phrases. Both as an argument and as a satellite the meaning of Causal expressions with a human referent can be classified in Homer in three subtypes within the continuum uniting the notions Cause and Agent: involuntary Cause, Responsible without Intention and Responsible with Intention.

\section{Introducción}

La Causa con referente humano presenta en Homero diversas expresiones cuyo uso parece estar condicionado tanto por factores sintácticos como semánticos. El presente trabajo tiene por objeto analizar, en primer lugar ${ }^{1}$, las formas empleadas en estrecha dependencia del verbo y determinar su significado. A continuación, se tratarán aquellas formas que admiten una interpretación como circunstantes o satélites, con el fin de constatar las posibles

* Éste es un trabajo realizado en el marco del Proyecto de Investigación PB 93-0254 (DGICYT). El análisis diacrónico de los datos y su conexión con la sintaxis indoeuropea se ha beneficiado de la ayuda de la Fundación Caja de Madrid. Expreso mi agradecimiento a J. L García Ramón, H. Hettrich y J. de la Villa por sus críticas y observaciones, así como a E. Crespo, con quien he discutido en detalle ésta y otras versiones anteriores.

1 En griego, al igual que en otras lenguas, las fronteras entre actantes o argumentos y circunstantes o satélites no son siempre fáciles de trazar. A pesar de ello, operaré con esta diferencia para facilitar el análisis de los datos (sobre esta cuestión, cf., entre otros, Vater 1978).

EMERITA (EM) - Revista de Lingüística y Filología Clásica - LXVII 2, 1999 - pp. 295 - 313 
coincidencias de uso respecto a las primeras. Dado que las funciones semánticas no se organizan en la lengua como parcelas conceptuales estancas, sino como nociones graduales que tienen algunos puntos de máxima diferenciación y otros de estrecho contacto, en el trabajo se intentará identificar la parcela significativa que ocupa en Homero cada una de las expresiones de causa con referente humano en el continuum entre las nociones de Causa y Agente $^{2}$.

\section{Empleo de expresiones de causa como actantes}

En los poemas homéricos, las expresiones de Causa se documentan como segundo complemento de un grupo reducido de verbos de sentimiento, que describen, bien el dolor o la aflicción del sujeto (Suj.), bien su cólera o irrita-

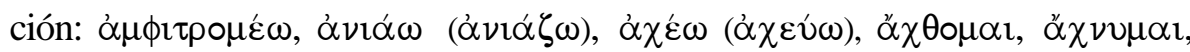

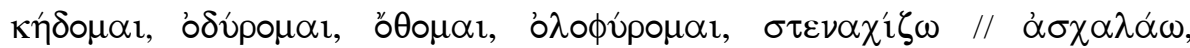

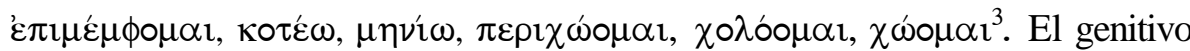
(Gen.) sin preposición, que en estos contextos designa tanto tanto entidades abstractas y objetos inanimados (1) como seres humanos (2), es la forma empleada con mayor frecuencia ${ }^{4}$ :

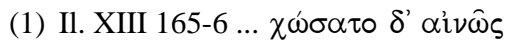

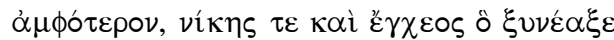

se encolerizó sobremanera (scil. Meríones) tanto por la victoria como por la pica, que se le había roto

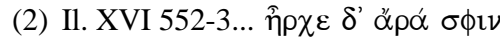

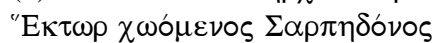

Héctor los guiaba, encolerizado por Sarpedón

2 La concepción de las funciones como nociones graduales, propuesta por la semántica de los prototipos, ha sido aplicada al estudio de la sintaxis casual de lenguas IE como el védico o el griego (cf. Hettrich 1995, Luraghi 1995 y 1996, Crespo 1997 y de la Villa 1998). La existencia de un eje o continuum conceptual entre las nociones de Causa y Agente en griego ha sido planteada por Luraghi 1996, Crespo 1997 y de la Villa 1998.

3 Para seleccionar los verbos se ha partido de la información de las gramáticas, que se ha completado con la que proporciona el diccionario inverso de Güthling (1913). La recopilación y clasificación del material se ha llevado a cabo con ayuda del CD-ROM del TLG.

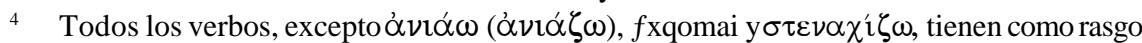
común el uso del Gen. sin preposición. Para simplificar el análisis de los datos, me voy a centrar en el grupo mayoritario de verbos, ya que los tres primeros no presentan peculiaridades de interés y se explican sin dificultad según el esquema que se propone para el resto; haré alusión a ellos en nota. 
Si bien estos Gen. se han considerado en ocasiones como expresiones partitivas $^{5}$, los poemas homéricos tan sólo aportan datos a favor de su interpretación como formas causales. Así, el hecho de que algunos de los verbos

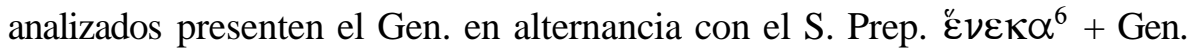
puede interpretarse como indicio de la función causal del primero. En los

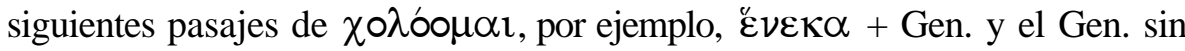
preposición se emplean con referentes inanimados haciendo alusión a nociones muy próximas ${ }^{7}$ :

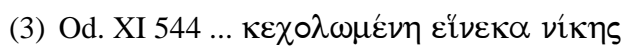
'irritada (scil. el alma de Ayante) por la victoria'

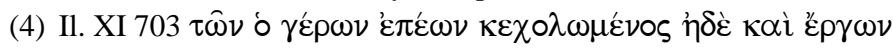
el anciano, irritado por estas palabras y obras, ...

La hipótesis de la función causal del Gen. se ve confirmada por la posibilidad de coordinar las formas de Gen. tanto con el S. Prep. cneka + Gen. (5) como con subordinadas causales $(6)^{8}$ :

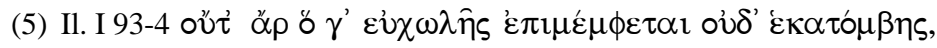

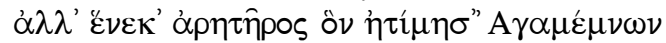
no está irritado (scil. Apolo) ni por una plegaria ni por una hecatombe, sino a causa del sacerdote al que ha deshonrado Agamenón

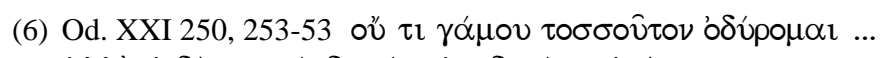

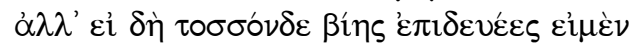

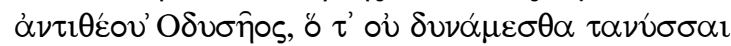
$\tau o ́ \xi o v . . .9$

5 Cf. Brugmann (1913, p. 443) y Lasso de la Vega (1968, p. 422-3). Menos clara es la postura de Schwyzer-Debrunner (1950: 133) y de Chantraine (1953: 56, 65), que creen plausible tanto la interpretación causal del Gen. como la partitiva. Adrados (1992, pp. 94, 153-4, 156, 160) niega el significado específico de este Gen. y lo equipara al llamado Ac. objeto; se trataría, en su

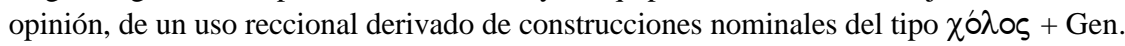

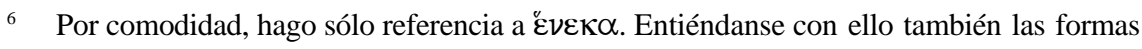

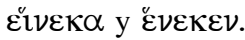

7 Con otros verbos, el Gen. se utiliza en alternacia con oraciones subordinadas que admiten

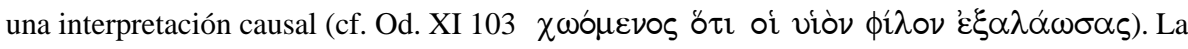
línea divisoria entre este tipo de oraciones y las completivas parece ser difusa (cf. SchwyzerDebrunner 1950, pp. 645-6, 662 o Chantraine 1953, p. 288). Para un análisis de ǒ $\tau$ c con verbos de sentimiento, cf. Muchnová (1989).

8 Sobre la coordinación como indicador de identidad funcional, cf. Dik (1968, p. 200 ss.). Para la aplicación de este criterio en griego, cf. Crespo 1988 y de la Villa 1989b.

9 En este caso no es posible atribuir a la subordinada un valor completivo. Por otra parte, el contexto parece excluir una interpretación condicional de $\alpha \lambda \lambda \lambda^{\prime}$ ' $\varepsilon \dot{\imath}$ : la superioridad de la fuerza 
no me lamento tanto por el matrimonio, sino porque somos en tal grado inferiores al divino Odiseo en fuerza que no somos capaces de tensar el arco

En todos los pasajes analizados, el uso del Gen. sin preposición coincide con la definición propuesta habitualmente para la Causa: entidad, situación o persona que provoca o posibilita un estado de cosas sin tener control sobre él ${ }^{10}$.

Cuando el referente es inanimado, el Gen. sin preposición se presenta en alternancia con $\check{\varepsilon}^{\prime} v \varepsilon \kappa \alpha+$ Gen. ${ }^{11},{ }^{\prime} \varepsilon \kappa+$ Gen. ${ }^{12}$ y con el Dat. sin preposición. El escaso uso del Dat. sin preposición, limitado al ejemplo de (7), es sorprendente, pues en el ámbito de la Causa el Dat. tiene en Homero una productividad mayor que el Gen., ya que no está limitado, como este último, a un uso como actante del verbo ${ }^{13}$ :

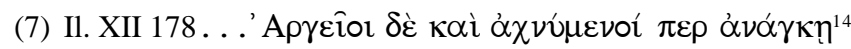

los argivos, aun disgustados por la necesidad...

En el caso de los referentes humanos, la situación es algo distinta: el Gen.

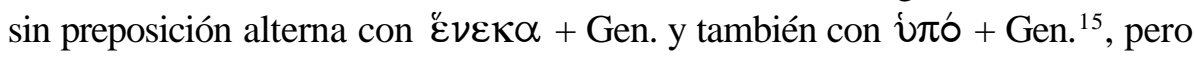

de Odiseo es ya una evidencia, pues tanto otros pretendientes como el propio Eurímaco, quien pronuncia estas palabras, han intentado sin éxito tensar el arco. El único que no ha probado sus fuerzas ha sido Antínoo.

10 Para la definición de Causa, cf. Vester 1983, p. 46 y Jackendoff 1990, p. 188), quien propone un concepto que engloba diversas funciones. El valor causal del Gen. con verbos de sentimiento es también defendida por Muchnová 1989, pp. 240-1 en un estudio centrado en Jenofonte.

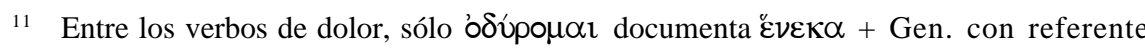

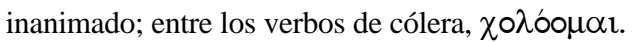

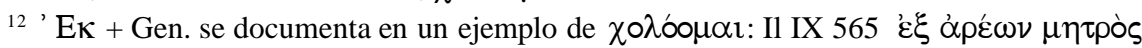
$\kappa \varepsilon \chi \circ \lambda \omega \mu \varepsilon \dot{\varepsilon} \circ \varsigma_{. . .}$

${ }^{13}$ A diferencia del Gen., el Dat. sin preposición se emplea con cierta frecuencia como circunstante para expresar la Causa con referentes inanimados (cf. Chantraine 1953, p. 77). El Dat. designa, por lo general, entidades abstractas.

14 El contenido léxico de $\alpha \nu \alpha ́ \alpha \kappa \eta$ permite también una interpretación del Dat. como Agente o Fuerza. El siguiente ejempo de $\alpha \chi \chi \theta o \mu \alpha \imath$ presenta una situación semejante: Il. V 354

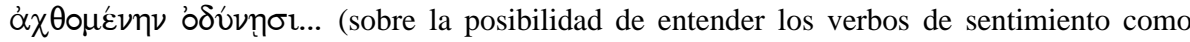
procesos o estados no controlados por el Suj. o como acciones controladas, cf. infra).

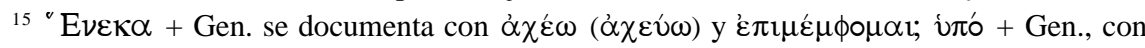
$\kappa \eta ́ \delta o \mu \alpha$ l. Entre los verbos que no presentan formas de Gen. sin preposición, $\sigma \tau \varepsilon v \alpha \chi i \zeta \omega$ ofrece

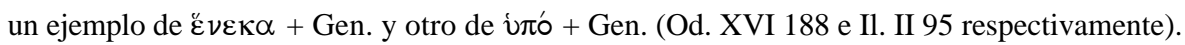


no con ' $\varepsilon \kappa+$ Gen.; por otra parte, no se documentan formas de Dat. como expresión de la Causa ${ }^{16}$.

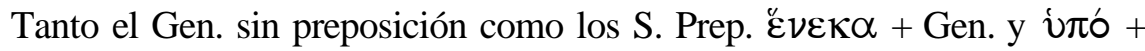
Gen. designan con estos verbos la persona que desencadena los sentimientos del Suj. sin ejercer control, al menos absoluto, sobre ellos. Sin embargo, la propia autonomía y capacidad de decisión que caracteriza al ser humano lo capacita para provocar un estado de cosas no sólo de forma involuntaria, sino también mediante una acción controlada que puede tener o no como fin el estado de cosas que se deriva de ella. En el caso concreto de los verbos de dolor y los verbos de cólera, el primer supuesto, que denominaré a partir de ahora Causante involuntario, aparece expresado mediante el Gen. sin prepo-

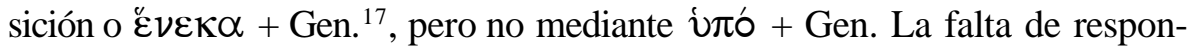
sabilidad del Causante involuntario sobre la situación que desencadena queda patente en numerosos ejemplos, ya que el Gen. sin preposición y $\check{\varepsilon} v \varepsilon \kappa \alpha+$ Gen. designan personas que carecen de toda capacidad de acción. De hecho, en muchas ocasiones son difuntos quienes provocan el dolor o la cólera de sus allegados o amigos:

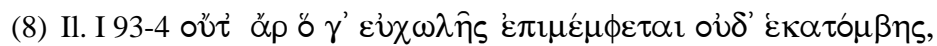

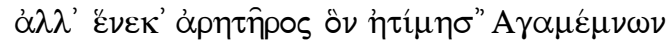
no está irritado (scil. Apolo) ni por una plegaria ni por una hecatombe, sino a causa del sacerdote al que ha deshonrado Agamenón

\footnotetext{
16 El Dat. que presentan algunos verbos de cólera ha de interpretarse como dativus incommodi. El siguiente pasaje muestra claramente cómo el Dat. y el Gen. desempeñan funciones

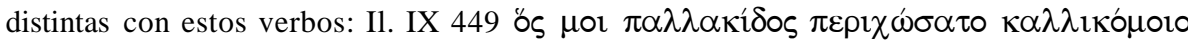
'quien (scil. mi padre) seirritó contra mí por una concubina, de hermosos cabellos'. Nos hallamos ante un antiguo Dat., cuya función originaria con referentes humanos fue, ya desde el IE, la expresión del Beneficiario.

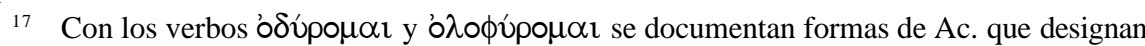

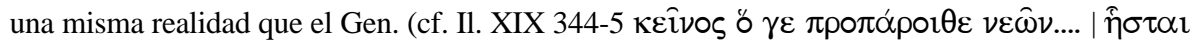

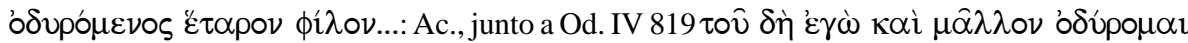

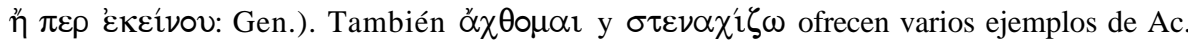
animado. Tanto la identidad referencial entre el Gen. y el Ac. con los dos primeros verbos como el propio contexto permite en todos los pasajes una interpretación del Ac. como expresión de la Causa. Ahora bien, puesto que la identidad referencial no implica necesariamente identidad funcional y el contexto no debe emplearse como criterio único, queda abierta la posibilidad de que el Ac. haya de entenderse como Paciente o Meta (Goal) (piénsese en español llorar por alguien / llorar a alguien). Sobre la pérdida del valor funcional del caso en formas adverbiales del tipo

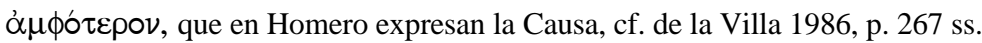




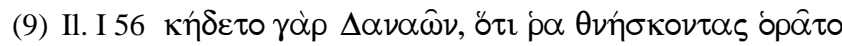
se inquietaba por los dánaos, ya que los veía morir

Cuando una persona provoca el estado de cosas descrito en la oración mediante una acción controlada, noción que a partir de ahora denominaré Responsable, pueden emplearse también el Gen. sin preposición (10) y

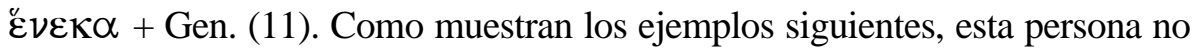
persigue con su acción controlada la situación que resulta de ella. Es decir, en estos casos el Responsable provoca los sentimientos del Suj. de forma no intencionada. Es interesante señalar que el Gen. sin preposición, documentado con frecuencia como expresión del Causante involuntario, sólo se emplea en el pasaje de (10) como designación del Responsable (Responsable sin intencionalidad $)^{18}$ :

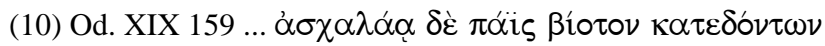
y se irrita el muchacho (scil. Telémaco) porque ellos están devorando su hacienda

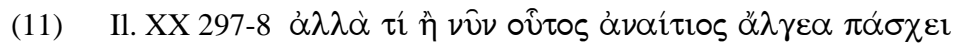

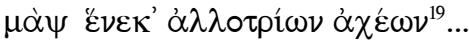
pero, ¿por qué este inocente (scil. Eneas) padece ahora dolores, sufriendo de forma inútil por culpa de otros?

La expresión de un Responsable que provoca intencionadamente los sentimientos del Suj. parece estar reservada para útó + Gen. Como se observa a continuación, el Responsable está ya muy próximo a un Agente $^{20}$ :

(12) Il. IV 497-8 ... viò $\delta \varepsilon \dot{~ T \rho \omega ̂ ع \varsigma ~ \kappa \varepsilon \kappa \alpha ́ \delta o v \tau o ~}$

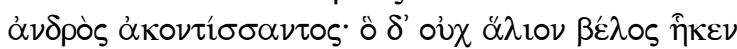

18 Téngase en cuenta que es el contexto el que permite inferir la presencia o ausencia de control e intencionalidad en la Causa con referentes humanos. Como se mostrará a continuación, este fenómeno tiene repercusiones formales en griego homérico.

19 El contexto nos informa de que nos hallamos ante Responsables, no ante Causantes

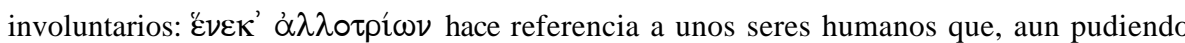
evitarlo, desencadenan el sufrimiento de Eneas con el descuido de sus obligaciones religiosas.

20 El control y la volición o intencionalidad son rasgos prototípicos del Agente (cf. DeLancey 1984 y Luraghi 1995). Estos rasgos, sin embargo, pueden darse separadamente en el Agente. La presencia del rasgo [+intencionalidad] implica o exige el rasgo [+control], de modo que todo Agente con intencionalidad tiene control sobre la acción. Por el contrario, que un Agente controle la acción no presupone que lo haga intencionadamente (cf. DeLancey 1984). Algunos autores, como el propio DeLancey, defienden que sólo se puede hablar de Agente cuando nos hallamos ante un Causante que controla intencionadamente la acción (un Causante con control, pero sin intencionalidad, no sería un Agente propiamente dicho). 
los troyanos se inquietaron por obra del varón que les disparó;

y no arrojó el proyectil en vano

Los datos de los verbos analizados permiten establecer tres subtipos de Causa con referente humano, que pueden definirse en los siguientes térmi$\operatorname{nos}^{21}$ :

a) Causante involuntario: persona que desencadena el estado de cosas descrito en la oración por medio de una situación que escapa a su control (cf. «dejó los estudios a causa de sus hijos, que eran todavía muy pequeños»).

b) Responsable sin intencionalidad: persona que desencadena el estado de cosas con una acción controlada, pero inintencionada (cf. «el presidente dimitió por culpa de los ministros corruptos de su gabinete»).

c) Responsable con intencionalidad: persona que desencadena el estado de cosas con una acción controlada e intencionada que tiene como fin el resultado que se deriva de ella. (cf. «aquel rey perdió el trono por culpa de un trai$d o r »)^{22}$. La diferencia entre el Responsable con intencionalidad y el Agente es, en numerosas ocasiones, mínima.

Los resultados obtenidos en el análisis de las expresiones de Causa en su uso como actantes de los verbos de dolor y los verbos de cólera se resumen en los siguientes puntos:

El Gen. sin preposición es la marca habitual de la Causa tanto para referentes inanimados como humanos. El Dat. sin preposición, por el contrario, sólo se emplea de forma ocasional con referentes inanimados, y nunca con referentes humanos.

Con referentes humanos, el Gen. sin preposición se emplea generalmente como expresión de lo que hemos denominado Causante involuntario. Sólo en un pasaje expresa el Responsable; se trata, con todo, de un Responsable sin intencionalidad. 1Eneka + Gen., menos frecuente que el Gen. sin preposición, puede expresar también una y otra noción. El Responsable con intencionalidad se expresa mediante $\mathfrak{\pi}$ ó + Gen.

\footnotetext{
21 También es posible establecer otros subtipos de Causa a partir de criteros diferentes de los que aquí se tienen en cuenta. Así, Vester 1983, p. 45, distingue la Causa propiamente dicha de la Razón en función de la ausencia o presencia de control por parte del Suj.

22 En español las expresiones gracias a y por culpa de parecen estar especializadas en la designación del Responsable; presentan, además, una valoración positiva o negativa de la acción de este Responsable.
} 
Esta situación puede entenderse como resultado del siguiente proceso: El antiguo ablativo (Abl.) IE fue la marca originaria de la Causa con los verbos de dolor y los verbos de cólera ${ }^{23}$. El antiguo Instrumental (Instr.), que tal vez compitió en un principio con aquél, se especializó con el tiempo como segundo complemento de los verbos de disfrute o alegría ${ }^{24}$; con los verbos de dolor y cólera, su uso quedó excluido en el caso de los referentes humanos y se

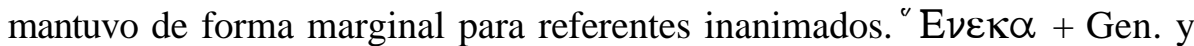
v̇ó + Gen., formas más recientes que el Gen. sin preposición, comenzaron a utilizarse ante la necesidad de recaracterizar preposicionalmente los casos heredados del IE; con todo, el funcionamiento del Gen. como actante permitió la continuidad de su uso sin preposición. Ahora bien, su espectro semántico se redujo: el morfema, que en su origen debió de expresar todo el continuum conceptual comprendido entre el causante involuntario y el Responsable con intencionalidad hasta la zona de confluencia de éste con el Agente, quedó relegado a la expresión del Causante involuntario y del Responsable

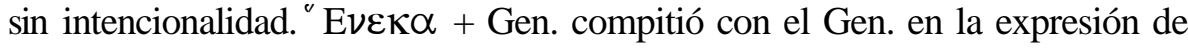
estas mismas nociones. La expresión del Responsable con intencionalidad, noción próxima al Agente, fue asumida por v̇ó + Gen., una de las marcas de esta función en Homero y en el griego posterior.

\section{Empleo de expresiones de Causa con referente humano como circunstantes}

El análisis de los verbos de dolor y los verbos de cólera permite obtener una visión parcial del funcionamiento de las expresiones de Causa con refe-

23 La comparación con otras lenguas IE permite suponer el uso del Abl. tanto con estos verbos como con los que expresan el temor del Suj. (Delbrück 1893, pp. 213-4).

24 Puesto que uno de los usos fundamentales del Instr. IE consistía en la expresión del instrumento del que se sirve un Agente para llevar a cabo una acción controlada, no es de extrañar que el Dat. griego se especializara como expresión del segundo complemento de los verbos de disfrute y alegría, que admiten un grado de control por parte del Suj. mayor que los verbos de dolor o cólera. A pesar de que todos los verbos de sentimiento pueden ser concebidos, en principio, como procesos (o estados) no controlados por el Suj. o como acciones controladas por él, (cf. Mumm 1996, pp. 48-51), unos verbos favorecen más la primera interpretación y otros la segunda. Así, el disfrute o la alegría se representan con frecuencia en la lengua como el resultado de una acción controlada y consciente del Suj. En Od. VI 104, por ejemplo, se muestra a la

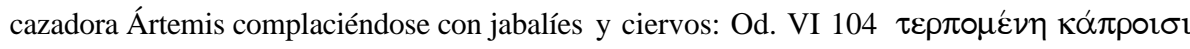

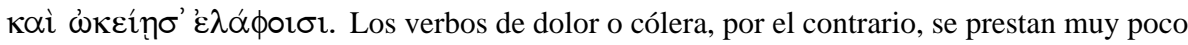
a una representación semejante de los sentimientos del Suj. 
rente humano en los poemas homéricos. Para completar esta visión parcial se ha de comprobar si la situación que presentan los verbos objeto de estudio tiene un carácter general o si responde a factores particulares y exclusivos de estos contextos. En este sentido se plantean dos cuestiones fundamentales: la productividad del Gen. y del Dat. sin preposición en su uso como circunstantes y la pertinencia de la distinción entre Causante involuntario, Responsable sin intencionalidad y Responsable con intencionalidad como nociones relevantes en la expresión de la Causa.

En lo que atañe a la productividad del Gen. y del Dat. sin preposición como expresiones de la Causa con referente humano, el análisis de los poemas homéricos ofrece los siguientes datos:

El empleo del Gen. sin preposición como circunstante es muy reducido ${ }^{25}$. Además, los ejemplos de que disponemos admiten siempre una interpretación alternativa. Se trata, como muestra el siguiente pasaje, de oraciones en las que se puede atribuir al Gen. tanto una función causal como un funcionamiento adnominaR ${ }^{6}$. Obsérvese que, de optarse por la primera interpretación, habríamos de tipificar el Gen. como expresión del Causante involuntario, valor que coincide con el que muestra el morfema en la práctica totalidad de los ejemplos de los verbos de dolor y los verbos de cólera:

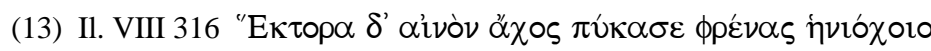
una atroz aflicción envolvió las mientes de Héctor a causa del auriga

El Dat. sin preposición, que en su uso como actante de los verbos de alegría podría interpretarse en ocasiones como expresión causa ${ }^{7}$, tampoco forma parte de las expresiones habituales de la Causa con referente humano en la esfera de los circunstantes ${ }^{28}$. Sólo he encontrado un ejemplo de estas características:

25 La situación parece ser semejante en el caso de los referentes inanimados y abstractos.

26 Estos sustantivos tienen la misma base léxica que los verbos analizados en el trabajo (cf. áxos).

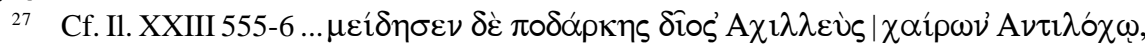

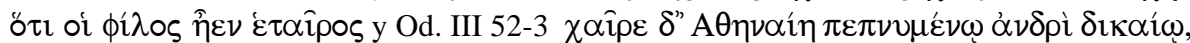

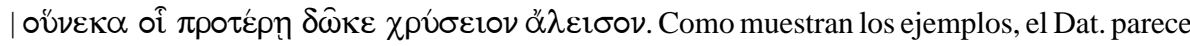
expresar en estos contextos tanto el Causante involuntario como el Responsable.

28 En época clásica, el Dat. sin preposición ha sido ya sustituido, sin excepciones, por $\mathrm{S}$. Prep. (cf. Luraghi 1989, pp. 301-2). 


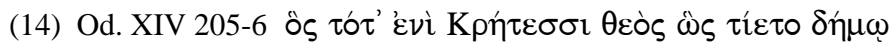

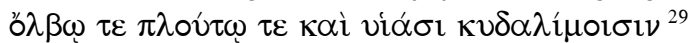
Quien (scil. Cástor) entre los cretenses era venerado por el pueblo como un dios por su dicha, su riqueza y por sus ilustres hijos

La conclusión es evidente: en su uso como circunstantes, los poemas homéricos sólo ofrecen escasos restos del uso del Gen. y del Dat. sin preposición como expresión de la Causa con referente humano. Las formas casuales han sido sustituidas por S. Prep.

Se hace necesario, por tanto, un análisis de estos S. Prep. que permita determinar si los tres subtipos de Causa establecidos encuentran en Homero un reflejo formal. Para ello se han seleccionado los ejemplos con referente humano de los S. Prep. que las gramáticas tipifican como expresiones de la

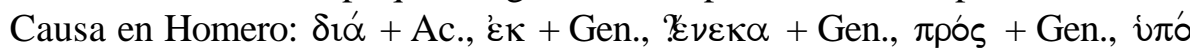
+ Gen. y íó + Dat. ${ }^{30}$ Los resultados obtenidos han sido los siguientes:

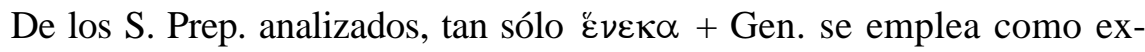
presión del Causante involuntario:

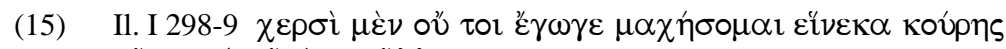

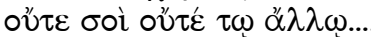

con mis manos yo no pienso luchar a causa de la muchacha (scil. Briseida) ni contigo ni con ningún otro

Frente a ello, todos los S. Prep. pueden expresar lo que hemos denomina-

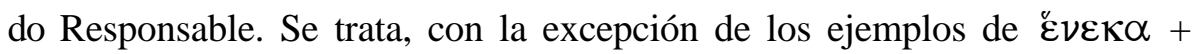
Gen. (cf. 16), de un Responsable con intencionalidad. Obsérvese que, salvo en el caso de (16), el Responsable tiene un control, mayor o menor, sobre la situación que desencadena. Así, en oraciones como las de (17) y (18), el Responsable instiga o fuerza al Suj. a llevar a cabo una acción verbal controlada por este último; en otras, como la de (19), el control del Suj. sobre la acción verbal parece muy reducido, lo que concede al Responsable un mayor protagonismo; en otras oraciones, por último, el Responsable provoca un proceso o estado sin voluntad ni control por parte del Suj. (cf. 20 y 21). Vemos, pues, cómo la distancia conceptual entre el Responsable y el Agente se acorta de forma progresiva. Dos son los factores que propician este hecho:

29 La información contextual no permite determinar ante qué tipo de Causante nos hallamos.

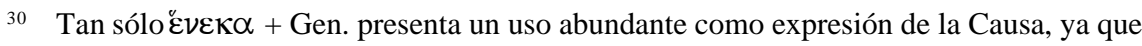
los ejemplos de $\delta \mathfrak{i} \alpha+$ Ac. referente humano son poco numerosos; por otra parte, ' $\varepsilon \kappa+$ Gen. y

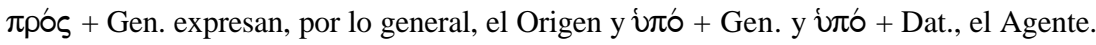


la intencionalidad del Responsable y su control creciente sobre el estado de cosas que desencadena; como muestran los ejemplos, el grado de control del Responsable aumenta según decrece el control del Suj. ${ }^{31}$ :

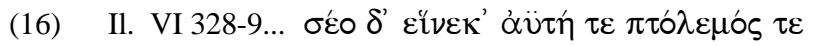

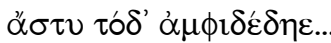

por tu culpa (scil. Alejandro) el grito de guerra y el combate arden en torno de esta ciudadela

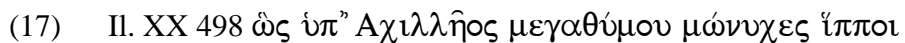

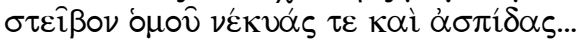

así, bajo las órdenes del magnánimo Aquiles pisoteaban los solípedos caballos cadáveres y escudos al tiempo

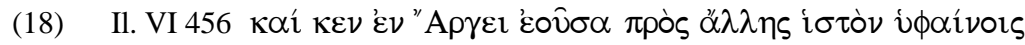
y quizás, estando en Argos, tejas la tela por orden de otra

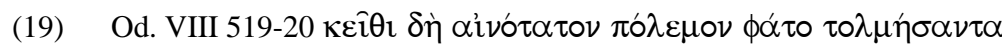

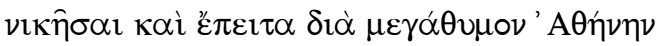
allí, dijo, se atrevió a trabar el más duro combate (scil. Odiseo) y venció al fin gracias a la magnánima Atena

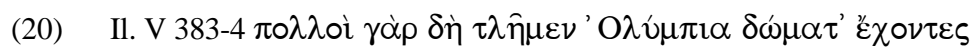
' $\xi \xi \alpha \nu \delta \rho \omega \hat{\nu} . . .32^{32}$

pues muchos de los que poseemos olímpicas moradas hemos sufrido por culpa de los hombres ...

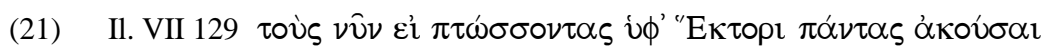
si se enterase (scil. Peleo) de que ahora todos ellos (scil. los argivos) se atemorizan por obra de Héctor

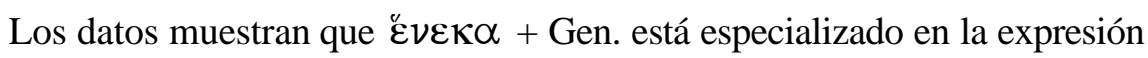
de las variantes conceptuales de la Causa más alejadas de la noción del Agente: el Causante involuntario y el Responsable sin intencionalidad. La información es menos precisa, sin embargo, en lo que atañe a $\delta i \alpha ́$ + Ac.; los escasos pasajes de que disponemos parecen indicar que el S. Prep. expresa el Responsable que actúa de forma intencionada, pero sin ejercer un control pleno sobre el estado de cosas descrito en la oración. La ausencia de ejemplos de $\delta i \alpha ́$ + Ac. como expresión del Causante indirecto no impide, con todo, suponer que este S. Prep. también ocupa una parcela conceptual relati-

31 DeLancey 1984 establece una gradación de agentividad en el sentido inverso: la "causa directa" (generalmente, un Agente prototípico) es menos agentiva en aquellos casos en los que actúa por medio de una "causa indirecta" (instrumento o causa propiamente dicha).

32 Son palabras de Dione, que consuela a Afrodita, quejumbrosa por las heridas que le ha causado Diomedes. A continuación, la diosa menciona otras afrentas de los hombres contra los inmortales. 
vamente alejada de la noción del Agente. De hecho, de los S. Prep. analiza-

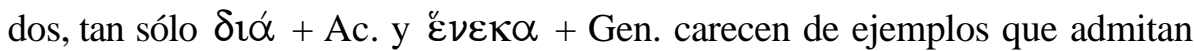
una interpretación como expresiones del Agente ${ }^{33}$. En el caso de ' $\varepsilon \kappa+$ Gen.,

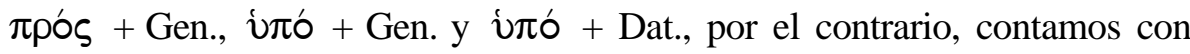
oraciones pasivas de carácter léxico o de carácter morfológico. Son estas últimas las que propician en mayor medida una interpretación de los S. Prep. en términos de Agente $^{34}$ :

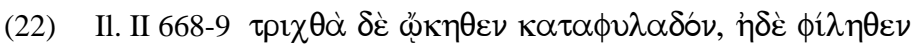
¿́k $\Delta$ tós ...

colonizaron la tierra divididos en tres tribus, y fueron amados por Zeus

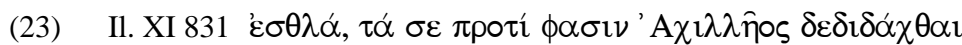
remedios que cuentan que te fueron enseñados por Aquiles

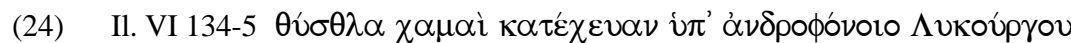

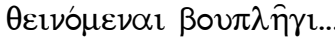

dejaron caer los tirsos al suelo (scil. las Ninfas), al ser golpeadas por el homicida Licurgo con la aguijada

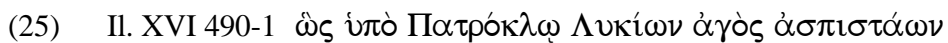
$\kappa \tau \varepsilon \imath v o ́ \mu \varepsilon v o \varsigma ~ \mu \varepsilon v \varepsilon ́ \alpha \imath v \varepsilon . .$.

así se enfurecía el capitán de los escudados licios, herido de muerte por Patroclo

Vemos, por tanto, cómo las expresiones de Causa con referente humano presentan un mismo significado en su uso como actantes y en su uso como circunstantes, si bien el empleo productivo del Gen. (y del Dat.) sin preposición se limita a su funcionamiento como actante. Así, la diferencia semántica entre el Causante involuntario, el Responsable sin intencionalidad y el Responsable con intencionalidad, establecida en los complementos de los verbos de dolor y los verbos de cólera, encuentra también reflejo formal en el caso de los circunstantes. Estas nociones forman parte de un continuum significativo y funcional cuyos extremos están definidos por el Causante involuntario y el Agente. Las diferentes expresiones de Causa con referente humano

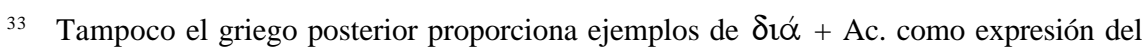
Agente, a pesar de que el S. Prep. se ha convertido ya en la expresión más habitual de la Causa. La información contextual muestra cómo incluso las oraciones con una pasiva morfológica se prestan poco a una interpretación de $\delta i \alpha ́$ + Ac. como Agente (al respecto, cf. Luraghi 1994, pp. 236-7).

34 En Homero sólo v́đó + Gen. y úđó + Dat. pueden considerarse marcas seguras del Agente prototípico (cf. de la Villa 1998, pp. 170-1). Obsérvese cómo en (22) y (23) el valor de

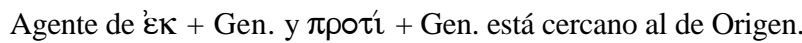


abarcan zonas concretas de este continuum, que en ocasiones coinciden total o parcialmente y que en otras, por el contrario, se complementan:

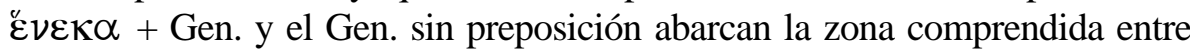
el Causante involuntario y el Responsable sin intencionalidad; $\delta \imath \alpha+$ Ac., la

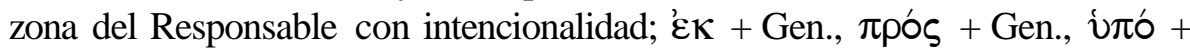

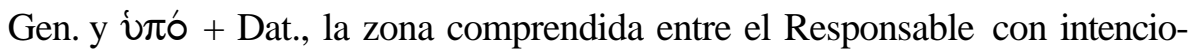
nalidad y el Agente.

El siguiente cuadro representa la distribución de las expresiones causales con referente humano en este continuиm ${ }^{35}$. Como puede apreciarse, las fronteras entre los distintos subtipos de Causa son difusas, pues los ámbitos de uso de cada una de las formas se superponen parcialmente:

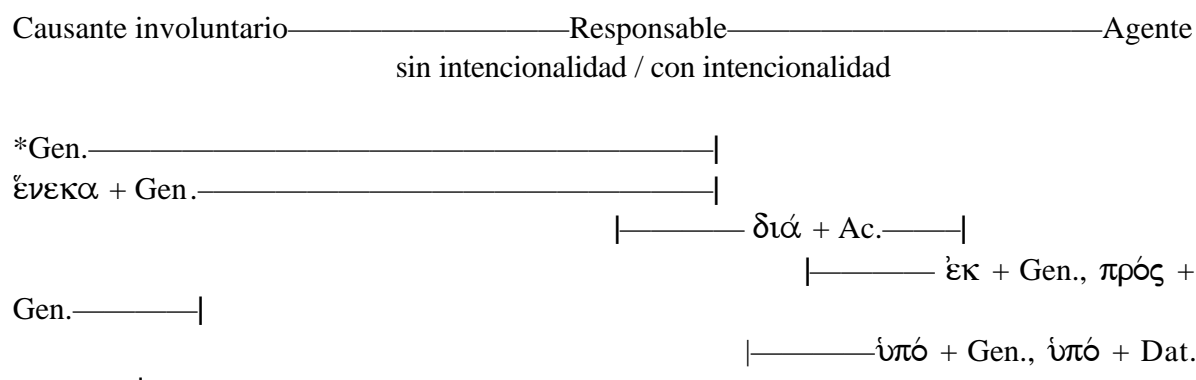

*Empleo productivo tan sólo en la esfera de los actantes.

La diferencia semántica y funcional entre el Causante involuntario y el Agente, puntos extremos del continuum, se hace patente en la yuxtaposición entre $\check{\varepsilon} \nu \varepsilon \kappa \alpha+$ Gen. y $\dot{v} \pi$ ó + Gen. de (26):

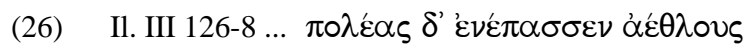

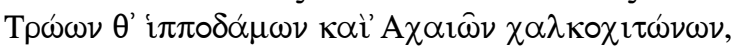

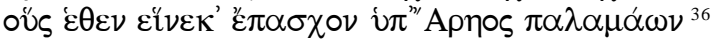

\footnotetext{
35 No he tenido en cuenta el Dat., ya que su estudio, que obligaría a plantearse las relaciones entre las nociones de Causa e Instrumento, supera los límites de este trabajo (sobre este punto, cf. de la Villa 1998).

36 Si bien los Agentes prototípicos son los seres humanos, las partes del cuerpo humano, las entidades abstractas y los objetos inanimados asociados claramente a ellos admiten con frecuencia una interpretación como Agentes.
} 
y bordaba (scil. Helena) numerosos enfrentamientos

de troyanos, domadores de caballos, y de aqueos,

de bróncineos mantos, que por su causa padecían a manos de Ares

Pasajes como éste demuestran que las formas situadas en el extremo izquierdo del continuum son incompatibles con la expresión de las nociones situadas en el extremo derecho, así como las situadas en el extremo derecho lo son con las nociones del extremo opuesto.

Hay, sin embargo, una posible excepción a este respecto: el supuesto uso del Gen. sin preposición como expresión del Agente. Esta hipótesis se basa

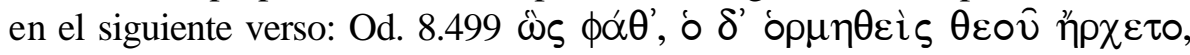

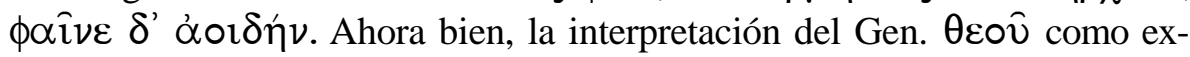
presión del Agente plantea algunas dificultades. En primer lugar, no hay argumentos suficientes para reconstruir un antiguo Abl., ya que en los poemas homéricos los S. Prep. de origen ablativo empleados como expresión del Agente sólo se documentan con formas personales del verbo o con infinitivos, pero no con participios ${ }^{37}$. De aceptarse el valor pasivo de óp $\mu \eta \theta \varepsilon i ́ \iota \varsigma$, parece más acertado interpretar $\theta \varepsilon \circ \hat{v}$ en términos de Gen. posesivo (Gen. auctoris $)^{38}$, ya que ésta es la construcción documentada en otras lenguas IE en el caso de participios pasivos y adjetivos verbales ${ }^{39}$. En segundo lugar, el propio valor pasivo del participio ó $\rho \eta \eta \theta \varepsilon i ́ \varsigma$ es dudoso, ya que en Homero las formas medio-pasivas de óp $\mu \alpha \dot{\omega} \omega$ con Suj. humano se emplean sin excepciones con un valor medio ${ }^{40}$. Además, si el Gen. $\theta \varepsilon \circ \hat{v}$ se hace depender de este participio, sea cual sea su función, se habrá de entender un uso absoluto de óp $\rho \chi \rho \mu \alpha 1$, anómalo en Homero cuando el verbo se emplea con el significado 'comenzar'"11.

37 Cf. Hettrich 1990, p. 93. El origen ablativo de $\theta \varepsilon \circ \hat{v}$ es defendido por Jamison 1979, p. 141. Chantraine 1953, p. 65, y Jankuhn 1969, p. 102 admiten también esta posibilidad, aunque con reservas.

38 Cf. Schwyzer 1943, p. 14, Schwyzer-Debrunner 1950, p. 119, Chantraine 1953, p. 61, Hettrich 1990, p. 93, y Adrados 1992, p. 131. Según estos autores, el valor de este Gen. sería idéntico, al menos en su origen, al de formas como la del siguiente pasaje: Il. I $273 \kappa \alpha i ̀ ~ \mu \varepsilon ́ \varepsilon \nu \mu \varepsilon v$

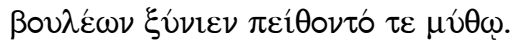

39 Sobre el uso del Gen. IE en estos contextos, cf., entre otros, Delbrïck 1893, p. 348, y Hettrich 1990, pp. 71-2, 92-4.

40 Ebeling 1885, s.u., propone para estas formas las traducciones surgo, ruo, curro e irruo.

41 Cf. Ebeling 1885, s.u.; véase también Anastasiou-Mader 1979, s.u. 
Parece más acertado, por tanto, plantear el valor medio de óp $\mu \eta \theta \varepsilon i ́ \varsigma$ y el uso de $\theta \varepsilon \circ \hat{v}$ en dependencia de la forma $\eta^{\prime} \rho \chi \varepsilon \tau \circ^{42}$. Según esta interpretación, el pasaje discutido describiría el momento en el que el aedo Demódoco, inspirado, comienza su canto con una invocación a la divinidad para narrar a continuación el episodio de la construcción del caballo de Troya ${ }^{43}$ :

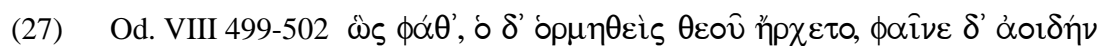

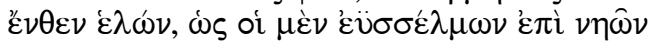

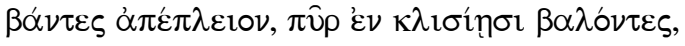
'Apreior...

así habló (scil. Odiseo) y aquél, inspirado, comenzó por la divinidad y mostró su canto, tomándolo desde el momento en que los argivos se hicieron a la mar en sus naves bien provistas de bancos una vez que incendiaron el campamento

Esta interpretación, que no presenta inconvenientes de orden sintáctico o semántico, encuentra también el apoyo en los Himnos homéricos, cuya fórmula inicial muestra en ocasiones un claro paralelismo con la construcción

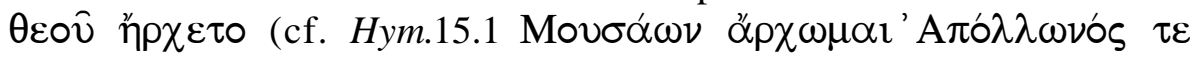

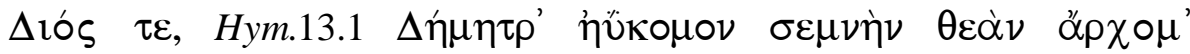
$\left.\alpha \varepsilon^{\prime} \delta \varepsilon \varepsilon \imath\right)^{44}$. Se ha de tener también en cuenta, por último, que de las tres interpretaciones del pasaje aquí esbozadas, ésta es la única mencionada en los escolios homéricos ${ }^{45}$.

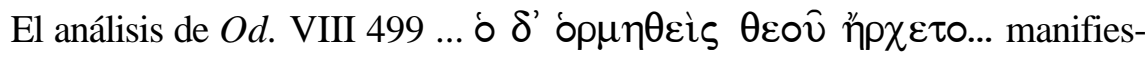
ta, a mi entender, la falta de datos que corroboren el empleo del Gen. homé-

42 Esta interpretación del texto es defendida, entre otros, por Calhoun 1938, pp. 205-6, Koster 1952, pp. 91-3, y Hainsworth 1982, p. 290. Los dos últimos aducen también cuestiones métricas a favor de la consideración de $\theta \varepsilon \circ \hat{\text { como complemento de } \eta \rho \chi \varepsilon \tau o, ~ y ~ n o ~ d e ~ o ́ p \mu \eta \theta \varepsilon i ́ s \varsigma . ~}$

43 La invocación a uno o varios dioses vinculados a la inspiración poética precedía por lo general a la recitación de un poema épico (cf. Wilamowitz 1916, p. 463 ss. y Adrados 1976, p. 112 ss.).

44 Interesantes resultan también los dos últimos versos de los himnos 5.y 9.: Hym. 5.292-3.:

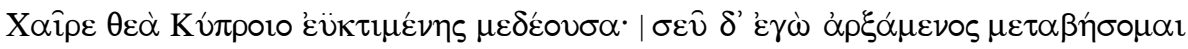

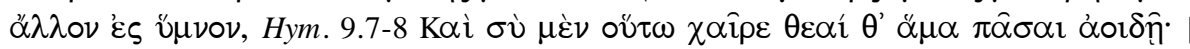

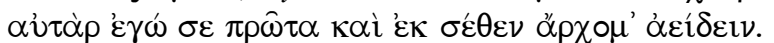

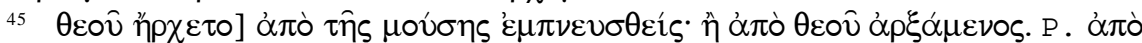

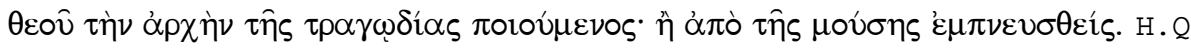
(Cito los escolios por la edición de Dindorf 1962). Junto a esta hipótesis, algunos escolios entienden también una relación entre óp $\mu \eta \theta \varepsilon i ́ \varsigma \varsigma$ y $\theta \varepsilon \circ \hat{v}$; en contra de los autores modernos, sin embargo, atribuyen al participio un valor medio y consideran el Gen. como expresión del Origen. 
rico sin preposición como expresión del Agente ${ }^{46}$. Por tanto, nada impide defender la incompatibilidad de las formas situadas en un extremo del continuum significativo Causa - Agente con las nociones del extremo contrario.

\section{Conclusiones.}

El Gen. sin preposición empleado con verbos de dolor y verbos de cólera expresa en los poemas homéricos la Causa. Junto al Gen. se documentan

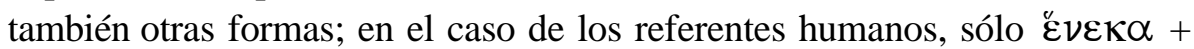
Gen. comparte la particularidades funcionales y semánticas del Gen. sin preposición.

Cuando el referente es humano, en Homero resulta pertinente establecer tres subtipos significativos de Causa, que encuentran reflejo formal tanto entre los complementos de los verbos de dolor y los verbos de cólera como entre los circunstantes de otros verbos: Causante involuntario, Responsable sin intencionalidad y Responsable con intencionalidad. El Gen. sin preposición y

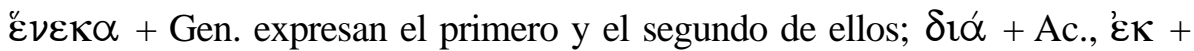

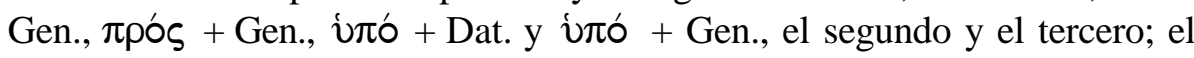

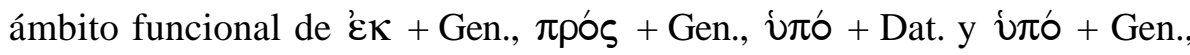
por su parte, se prolonga hasta la expresión del Agente. Todas estas nociones forman parte de un continuum significativo y funcional, cuyos extremos están definidos por el Causante involuntario y el Agente. Las formas que ocupan un extremo del continuum son incompatibles con la expresión de las nociones situadas en el extremo opuesto. Este hecho explica, por ejemplo, que el Gen. sin preposición no se emplee como expresión del Agente y que un S. Prep. como v̇ ó + Gen. tan sólo designe las variantes de la Causa más próximas al Agente.

El Gen. sin preposición, continuación del antiguo Abl. IE, es una antigua expresión causal con la que, poco a poco, comenzaron a competir formas

46 El Dat. sin preposición también se ha interpretado con frecuencia como una de las

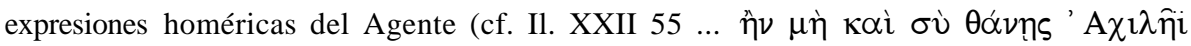
$\delta \alpha \mu \alpha \sigma \theta \varepsilon i \varsigma \varsigma$ ). Dado que este morfema apenas se emplea en Homero como expresión de la Causa con referente humano, no parece aconsejable poner en relación ejemplos como éste con un uso causal. El empleo en las estructuras pasivas del antiguo Instrumental para referentes inanimados pudo propiciar la extensión de este caso a referentes animados (Hettrich 1990, pp. 76-9). Es probable, por tanto, que nos hallemos ante una manifestación de la proximidad entre las nociones de Instrumento y Agente (cf. de la Villa 1998, pp. 157-9). 
con recaracterización preposicional; el uso del morfema casual sin preposición se fue limitando, con el tiempo, a un funcionamiento como actante de los verbos de dolor y los verbos de cólera. Como resultado de este empleo progresivo de S. Prep., el espectro semántico del Gen. sin preposición se redujo: el morfema, que en su origen debió de expresar todo el continuum conceptual comprendido entre el causante involuntario y el Responsable con intencionalidad (incluyendo el Agente), ha quedado relegado en Homero a la expresión del Causante involuntario y, en menor medida, del Responsable sin intencionalidad.

\section{REFERENCIAS BIBLIOGRÁFICAS}

Adrados, F. R. (1976): Orígenes de la lírica griega, Madrid.

(1992): Nueva sintaxis del griego antiguo, Madrid.

Anastasiou, J.-Mader, B. (1979): en Lexikon des frühgriechischen Epos (LfrgE), s.u. óp $\chi \omega$, Bd. 1. A, Göttingen.

Brugmann, K. (1913): Griechische Grammatik II, München.

Calhoun, G. M. (1938): «The poet and the Muses in Homer», Classical Philology 33, pp. 157-66

Crespo, E. (1988): «The semantic and syntactic functions of the Accusative», en A. Rijksbaron et al. (ed.) Proceedings in Commemoration of the 150th Anniversary of the Publication of Raphael Kühner's 'Ausführliche Grammatik der griechischen Sprache, II Teil: Syntax', Amsterdam, pp. 219-36.

(1997): «Sintaxis de los elementos de relación en griego clásico», Actas del IX Con-

greso de Estudios Clásicos, Madrid, pp. 3-42.

Chantraine, P. (1953): Grammaire homérique II: Syntaxe, París.

DeLancey, S. (1984): «Notes on agentivity and causation», Studies in Language 8, pp. 181214.

Delbrück, B. (1893): Grundriß der vergleichenden Grammatik der indogermanischen Sprachen III, $\quad 1$, Estrasburgo.

Dik, S. C. (1968): Coordination. Its Implications for the Theory of General Linguistics, Amsterdam.

Dindorf, G. (1962): Scholia Graeca in Homeri Odysseam, Amsterdam (= Oxford, 1855).

Ebeling, H. (1885): Lexicon homericum I, II, Leipzig.

García Ramón, J.L. (1998): «Adverbios de dirección e instrumental indoeuropeo», en E.Crespo y García Ramón (ed.) Actas del Coloquio de la Indogermanische Gesellschaft. Berthold Delbrück y la sintaxis indoeuropea hoy, Madrid, pp. 113-44. 
Güthling, O. (1913): Langenscheidts Großwörterbuch Deutsch-Altgriechisch, Berlín-Münich. Hainswort, J. B.- Privitera, G. A. (1982): Scrittori greci e latini. Omero, Odissea, volume II (Libri V- VIII), Venecia.

Hettrich, H. (1990): Der Agens in passivischen Sätzen altindogermanischer Sprachen, Nachrichten der Akademie der Wissenschaften in Göttingen, I.Philologisch-historische Klasse, Göttingen.

------ (1995): «Zur funktionalen Variationsbreite altindogermanischer Kasus: Der Ablativ im Rgveda», en H. Hettrich et al. (ed.) Verba et structurae, Festschrift für Klaus Strunk zum 65. Geburtstag, Innsbruck.

Jackendoff, R. (1990): Semantic Structures, Cambridge.

Jamison, S. (1979): «The Case of Agent in Indo-European», Sprache 25, pp. 129-43.

Jankuhn, H. (1969): Die passive Bedeutung medialer Formen untersucht an der Sprache Homers, Göttingen.

Kleiber, G. (1995): La semántica de los prototipos. Categoría y sentido léxico, Madrid.

Koster, W.J.W. (1952): «De graecorum genitivo, qui dicitur auctoris: de Od. $\theta$ 499», Mnemosyne 5, pp. 89-93.

Kühner, R. - Gerth, B. (1898): Ausführliche Grammatik der griechischen Sprache, II: Syntax, Hannover-Leipzig.

Latacz, J. (1966): Zum Wortfeld "Freude" in der Sprache Homers, Heidelberg.

Lasso de la Vega, J. L. (1968): Sintaxis griega I, Madrid.

Luraghi, S. (1989): «Cause and Instrument expressions in Classical Greek. Remarks on the use of $\delta 1 \alpha$ in Herodotus and Plato», Mnemosyne 43, pp. 294-308.

----- (1994): «Animate Nouns in Cause Expressions», en B. Jacquinod (ed.) Actes du colloque in ternational de Saint-Étienne 'Cas et prépositions en grec ancien', Saint-Étienne, pp. 227-37.

------ (1995): «Prototypicality and agenthood in Indo-European», en H. Andersen (ed.) Historical Linguistics 1993, Amsterdam, pp. 259-68.

(1996): Studi su casi e preposizioni nel greco antico, Milano.

Muchnová, D. (1989): «Analyse sémantico-syntaxique des propositions par ǒ $\tau \imath$ après les verba affectuum», Graecolatina Pragensia 12, 33-50.

- (1994): «Remarques sur les syntagmes prépositionnels et l'expression de la cause chez Xénophon», en B. Jacquinod (ed.) Actes du colloque international de SaintÉtienne 'Cas et prépositions en grec ancien', Saint-Étienne, pp. 239-48.

Mumm, P.-A. (1996): Parameter des einfachen Satzes aus funktionaler Sicht, Abriß ihrer onomasio logischen Systematik. Teil I: Relationierung der Lexeme in der Prädikation; Valenz, Numeralität und Aspektualität des Verbs, München.

La Roche, J. (1861): «Beobachtungen über den Gebrauch von úó bei Homer», Zeitschrift für die ös terreichischen Gymnasien, 12.Jg., pp. 337-77.

Schlesinger, I. M. (1989): «Instruments as agents: on the nature of semantic relations», Journal of Linguistics 25, pp. 189-210.

Schwyzer, E. (1943): «Zum persönlichen Agens beim Passiv, besonders im Griechischen», Abh.d. Preuß. Akad.d. Wiss., Phil.-hist. Kl., Berlin (=Kleine Schriften, Hrg. R. Schmitt, Innsbruck, pp. 3-79). 
Schwyzer, E. - Debrunner, A. (1950): Griechische Grammatik, II, München.

Vater, H. (1978): «On the possibility of distinguishing between complements and adjuncts», en W. Abraham (ed.) Valence, Semantic Case and Grammatical Relations, Amsterdam, pp. 21-45.

Vester, E. (1983): Instrument and Manner in Latin, Assen.

de la Villa, J. (1986): La sintaxis de los adverbios griegos, tesis doctoral inédita, Madrid.

(1989a): «Caractérisation fonctionnelle du datif grec», Glotta 67, pp. 20-40.

(1989b): «Las funciones de los elementos nominales: Criterios para su identificación en Griego y Latín», CFC 22, pp. 291-303.

(1998): «La agentividad en la lengua homérica», en M. E. Torrego (ed.) Nombres y fun ciones: Estudios de sintaxis griega y latina, pp. 147-80.

Wackernagel, J. (1926): Vorlesungen über Syntax, I , Basilea: Birkhäuser. von Wilamowitz-Moellendorf, U. (1916): Die Ilias und Homer, Berlin. 